\title{
Metabolic Mechanisms and a Rational Combinational Application of Carboxyamidotriazole in Fighting Pancreatic Cancer Progression after Chemotherapy
}

\author{
Rui Ju, ${ }^{1}$ Kailun Fei, ${ }^{1}$ Siang Li, Chen Chen, Lei Zhu, Juan Li, Dechang Zhang, Lei Guo, \\ and Caiying Ye
}

Department of Pharmacology, Institute of Basic Medical Sciences Chinese Academy of Medical Sciences, School of Basic Medicine Peking Union Medical College, Beijing, China

Received March 23, 2018; accepted July 11, 2018

\begin{abstract}
The anticancer and anti-inflammatory effects of carboxyamidotriazole (CAl) have been demonstrated in several studies, but the underlying mechanisms remain to be elucidated. This study showed that CAl caused metabolic reprogramming of pancreatic cancer cells. The inhibition of mitochondrial oxidative metabolism by $\mathrm{CAl}$ led to increased glutamine-dependent reductive carboxylation and enhanced glycolytic metabolism. The presence of environmental substances that affect cellular metabolism, such as glutamine and pyruvate, attenuated the anticancer efficacy of CAl. Based on the action of CAl: 1) when glutamine was removed, the NAD+/NADH ratio was decreased, the synthesis of cellular aspartate was reduced, and autophagy flux was blocked; and 2) when glycolysis was pharmacologically inhibited, the ATP level was significantly decreased, the cell

viability was greatly inhibited, and the compensatory rescue effect of glutamine was eliminated. When combined with chemotherapy, cotreatment with $\mathrm{CAl}$ and the glycolysis inhibitor 2-deoxyglucose (2-DG) inhibited the pancreatic cancer progression after chemotherapy. As the inhibition of mitochondrial oxidative metabolism can explain several anticancer activities of CAl reported previously, including inhibition of calcium entry and induction of reactive oxygen species, we demonstrate that inhibition of mitochondrial oxidative phosphorylation may be the fundamental mechanism of CAl. The combination of CAl and 2-DG causes energy depletion in cancer cells, eliminating the rescue effect of the metabolic environment. Inhibiting pancreatic cancer progression after chemotherapy is a rational application of this metabolism-disturbing combination strategy.
\end{abstract}

\section{Introduction}

Carboxyamidotriazole (CAI) is a small-molecule drug possessing anticancer activities (Felder et al., 1991; Kohn et al., 1992; Wasilenko et al., 1996; Luzzi et al., 1998). The mechanisms of these activities have been considered to be the inhibition of capacitative calcium entry or the promotion of reactive oxygen species (ROS) production (Mignen et al., 2005). Mignen et al. suggested that the main mechanism for CAI inhibiting cell proliferation was attributed to the inhibition of the mitochondrial membrane potential and capacitative calcium entry (Mignen et al., 2005; Corrado et al., 2011). CAI was also reported to promote ROS production and induce apoptosis in cancer cells. In a previous study, we found that

This work was supported by the National Natural Science Foundation of China [81672966 and 81402943], National Science and Technology Major Project [2014ZX09507003-003], and Chinese Academy of Medical Sciences Innovation Fund for Medical Sciences [2016-I2M-1-011 and 2016-I2M-1-002].

${ }^{1}$ R.J. and K.F. contributed equally to this work.

https://doi.org/10.1124/jpet.118.249326. the combination of CAI and other drugs that promote ROS production significantly increased the proportion of apoptotic cancer cells (Chen et al., 2017). These findings suggested the association between CAI and mitochondria.

We have preliminarily found that CAI can influence mitochondrial respiration in a variety of cancer cells. Bioenergetic tests have shown that it downregulates mitochondrial oxygen consumption in cancer cells and has a direct inhibitory effect on the activity of mitochondrial complex I on the respiratory chain (Ju et al., 2016). In recent years, the effect of mitochondrial respiration on cell proliferation and autophagy has been revealed. Studies have shown that the mitochondrial respiratory chain not only provides energy, but also supports aspartate synthesis in proliferating cells (Birsoy et al., 2015; Sullivan et al., 2015). In addition, respiratory chain defects will directly lead to the block of autophagy flux in $\mathrm{T}$ cells (Baixauli et al., 2015). These effects are all associated with the regulation of $\mathrm{NAD}+/ \mathrm{NADH}$ by the respiratory chain. It has been reported that environmental substances that affect cellular metabolism, such as pyruvate, which also regulates

ABBREVIATIONS: AKB, $\alpha$-ketobutyrate; CAI, carboxyamidotriazole; CSC, cancer stem cell; 2-DG, 2-deoxyglucose; DMEM, Dulbecco's modified Eagle's medium; ECAR, extracellular acidification rate; ETC, electron transport chain; FCCP, carbonyl cyanide-4 (trifluoromethoxy) phenylhydrazone; GEM, gemcitabine; GFP, green fluorescent protein; i.g., intragastrically; $\alpha$-KG, $\alpha$-ketoglutarate; OCR, oxygen consumption rate; OXA, sodium oxamate; OXPHOS, oxidative phosphorylation; PBS, phosphate-buffered saline; PEG400, polyethylene glycol 400; PEPCK, phosphoenolpyruvate carboxykinase; RFP, red fluorescent protein; ROS, reactive oxygen species. 
$\mathrm{NAD}+/ \mathrm{NADH}$, can dictate cancer sensitivity to drugs that impact cell metabolism (Gui et al., 2016). In addition, when oxidative phosphorylation (OXPHOS) is defective, the cells are supplied with energy, and the tricarboxylic acid cycle intermediates by enhancing glycolysis and reductive carboxylation fueled by glucose and glutamine (Mullen et al., 2011; Ward and Thompson, 2012; Du et al., 2016). Whether CAI exerts anticancer action by blocking cellular aspartate synthesis or autophagy flux, and whether the environmental metabolic supplies can influence the anticancer efficacy of CAI remain to be clarified.

Pancreatic cancer is one of the most lethal malignancies in the world. Gemcitabine is a nucleotide analog widely used in cancer treatment. It is currently the first-line chemotherapeutic drug for pancreatic cancer patients (Shewach and Lawrence, 1996; Burris et al., 1997; Louvet et al., 2005). However, resistance to gemcitabine can develop after repeated exposure, and the improvement in patients' long-term outcome is very unsatisfying.

We hypothesize that if CAI is applied to the cancer cell types or subpopulations that are more dependent on mitochondrial respiration, or combined with other metabolism-disturbing drugs (Ben Sahra et al., 2010; Cheong et al., 2011; LeBleu et al., 2014; Caino et al., 2015; Ansó et al., 2017; Frattini et al., 2018), it may exert much higher anticancer efficacy. In several studies, pancreatic cancer stem cells (CSCs) have been considered to be highly dependent on OXPHOS (Viale et al., 2014; Sancho et al., 2015). Elimination of highly chemoresistant CSCs via inhibition of mitochondrial function (or combined with glycolysis inhibitors) may effectively prevent pancreatic cancer relapse and thus improve patients' long-term outcome. Therefore, we speculated that CAI (or CAI combined with glycolysis inhibitors) may be useful in maintaining the efficacy of chemotherapies to prevent pancreatic cancer progression.

In this study, we investigated the effects of CAI on cancer cell metabolism and the environmental metabolic factors that affect its anticancer efficacy. We also found a better therapeutic strategy to optimize the application of the metabolismdisturbing drugs in fighting pancreatic cancer.

\section{Materials and Methods}

Cell Lines and Reagents. Pancreatic carcinoma Panc-1 and Pan02 cells were obtained from the Institute of Basic Medical Sciences Chinese Academy of Medical Sciences (Beijing, China). The cells grew in Dulbecco's modified Eagle's medium (DMEM; high glucose) supplemented with $10 \%$ fetal bovine serum, $50 \mathrm{mg} / \mathrm{ml}$ penicillin, $100 \mathrm{mg} / \mathrm{ml}$ streptomycin, and $4 \mathrm{mM}$ L-glutamine at $37^{\circ} \mathrm{C}$ in a humidified $95 \%$ $\mathrm{O}_{2}-5 \% \mathrm{CO}_{2}$ atmosphere. In some specific experiments, the culture medium was not supplemented with L-glutamine.

CAI was synthesized (purity $>99.0 \%$ ) by the Institute of Materia Medica, Chinese Academy of Medical Sciences (Beijing, China). Polyethylene glycol 400 (PEG400) was purchased from YIPUSHENG Pharmaceutical Co., LTD (Jiangxi, China). Gemcitabine hydrochloride and chloroquine diphosphate were purchased from MedChem Express (Princeton, NJ). 2-Deoxyglucose (2-DG) and sodium oxamate (OXA) were purchased from Sigma-Aldrich (St. Louis, MO).

Animals. BALB/c nude female mice and C57BL/6 female mice $(18-22 \mathrm{~g})$ were obtained from the Institute of Laboratory Animal Sciences, Chinese Academy of Medical Sciences. They were housed in an air-conditioned room $\left(22 \pm 2{ }^{\circ} \mathrm{C}\right.$ and $40 \%-70 \%$ humidity) with a controlled 12-hour light/dark cycle. Animals had free access to standard chow and water. The animal studies and procedures were approved by the Institutional Animal Care and Use Committee of Peking Union Medical College.
Determination of Oxygen Consumption Rate and Extracellular Acidification Rate. Oxygen consumption rate (OCR) in Panc-1 was measured with the Seahorse XF cell mito stress test kit (103015100; Agilent Technologies, Santa Clara, CA). The cells were cultured with minimally buffered DMEM (supplemented with $1 \mathrm{mM}$ pyruvate, $2 \mathrm{mM}$ glutamine, and $10 \mathrm{mM}$ glucose; $\mathrm{pH} \mathrm{7.4)}$ and allowed to equilibrate for 1 hour before measuring OCR. OCRs were measured under basal conditions and in response to $\mathrm{CAI}(0.5,1,2$, or $4 \mu \mathrm{M})$, oligomycin $(1 \mu \mathrm{M})$, carbonyl cyanide-4 (trifluoromethoxy) phenylhydrazone (FCCP; $0.5 \mu \mathrm{M}$ ), and rotenone plus antimycin $\mathrm{A}(0.5 \mu \mathrm{M})$. In another experiment, the cells were transferred onto XF microplates and treated with $10 \mu \mathrm{M}$ CAI for 24 hours before the OCR measurement.

Extracellular acidification rate (ECAR) in Panc-1 was measured with the Seahorse XF glycolysis stress test kit (103020-100; Agilent Technologies). The cells were cultured with minimally buffered DMEM (supplemented with $1 \mathrm{mM}$ glutamine; $\mathrm{pH}$ 7.4) and allowed to equilibrate for 1 hour before measuring ECAR. ECARs were measured under basal conditions and in response to $\mathrm{CAI}(0.5,1,2$, or $4 \mu \mathrm{M})$, glucose $(10 \mathrm{mM})$, oligomycin $(1 \mu \mathrm{M})$, and 2-DG (50 mM).

Oligomycin inhibits ATP synthase (complex V) and shifts the energy production to glycolysis. The decrease in OCR following injection of oligomycin correlates to the mitochondrial respiration associated with cellular ATP production, and increase in ECAR reveals the cellular maximum glycolytic capacity. FCCP is an uncoupling agent that collapses the proton gradient and disrupts the mitochondrial membrane potential. As a result, electron flow through the electron transport chain (ETC) is uninhibited and oxygen is maximally consumed by complex IV. The FCCP-stimulated OCR can then be used to calculate spare respiratory capacity, defined as the difference between maximal respiration and basal respiration. The third injection is a mix of rotenone, a complex I inhibitor, and antimycin A, a complex III inhibitor. This combination shuts down mitochondrial respiration and enables the calculation of nonmitochondrial respiration driven by processes outside the mitochondria.

Determination of NAD+/NADH Ratio. Following treatment with CAI for 48 hours, Panc-1 cells were divided equally into two tubes. The concentration of NAD + or NADH was then determined with the NAD+/NADH assay kit (E2ND-100; BioAssay Systems, Hayward, CA). Cells were washed in ice-cold phosphate-buffered saline (PBS) and homogenized with either $100 \mu \mathrm{l}$ of NAD extraction buffer for NAD determination or $100 \mu \mathrm{l}$ of NADH extraction buffer for $\mathrm{NADH}$ determination. The extracts were heated at $60^{\circ} \mathrm{C}$ for 5 minutes, and $20 \mu \mathrm{l}$ of assay buffer and $100 \mu \mathrm{l}$ of the opposite extraction buffer were added to neutralize the extracts. The samples were vortexed and centrifuged at 14,000 rpm for 5 minutes. For determination, $40 \mu \mathrm{l}$ of supernatant or NAD standards and $80 \mu \mathrm{l}$ of working reagent (containing assay buffer, enzyme A, enzyme B, lactate, and MTT) were added to a clear flat-bottom 96 -well plate. The optical density for time zero at $565 \mathrm{~nm}$ and the optical density after a 15-minute incubation at room temperature were recorded.

Cell Viability. Cell viability was calculated as the number of viable cells divided by the total number of cells within the grids on the hemacytometer. If cells take up trypan blue, they are considered nonviable. Stock solution of trypan blue $[0.4 \%$ (v/v, 1:10)] was prepared in PBS ( $\mathrm{pH} 7.2$ ) and added to cells. The cell suspension was loaded onto a hemacytometer and examined immediately under a microscope. The number of blue staining cells and total cells were counted.

Aspartate Level. Panc- 1 cells were seeded at $5 \times 10^{5}$ cells/dish in 6 -cm dishes overnight. On the following day, cells were washed twice in PBS and cultured in the medium containing the indicated treatments. After 24 hours, polar metabolites were extracted using $80 \%$ methanol in water. Soluble content was dried under nitrogen gas. The derivatized samples were analyzed by high-performance liquid chromatography-tandem mass spectrometry [Shimadzu (Kyoto, Japan) LC20AD high-performance liquid chromatography interfaced with an API 3200MD TRAP mass spectrometer].

Western Blotting. Western blot analysis was performed on lysates of Panc-1 cells after the indicated treatments. Cells were lysed on ice with 
Radio Immunoprecipitation Assay lysis buffer added with cOmplete Protease Inhibitor Cocktail (11697498001; Roche, Basel, Switzerland). Lysates were immunoblotted with the desired primary antibody for 2 hours at the following dilutions: anti- $\beta$-actin 1:5000 (A2228; SigmaAldrich), anti-LC3B 1:500 (L7543; Sigma-Aldrich), anti-p62 1:500 (P0067; Sigma-Aldrich), anti-NDUFS7 (AW5221; Abgent, San Diego, CA), anti-NDUFV3 (13430-1-AP; Proteintech, Rosemont, IL), anti-IDH1 (AW5173; Abgent), and anti-IDH2 (15932-1-AP; Proteintech). The membrane was incubated subsequently with the appropriate secondary antibody, and the immunoreactive protein bands were visualized using an enhanced chemiluminescence kit (Millipore Corporation, Billerica, MA) according to the manufacturer's instructions. All of the proteins were detected by a Tanon (Shanghai, China) Chemiluminescent Imaging System. The density ratio of a specific band over $\beta$-actin was determined with Quantity One software (Bio-Rad Laboratories, Hercules, CA).

Autophagy Flux. Panc- 1 cells were seeded at $1 \times 10^{4}$ cells/well in Nunc Laboratory-Tek II chambered coverglass systems (Thermo Fisher Scientific, Waltham, MA). Cells were infected with Green fluorescent protein - red fluorescent protein - LC3 (GFP-RFP-LC3 adenoviral particles (HB-AP210 0001; HanBio, Shanghai, China) for 4 hours; after infection, the cells were cultured for another 24 hours with the indicated treatments. Imaging was performed on an UltraVIEW VoX 3D Live Cell Imaging System (PerkinElmer, Inc, Waltham, MA). All image acquisition settings were kept at the same state during the image collection. Image analysis was performed using the Volocity Demo 5.4 software (PerkinElmer, Inc).

ATP Content. Following the indicated treatments, Panc-1 cells were lysed, and the ATP content in the supernatant was measured with the ATP assay kit (S0026; Beyotime Biotechnology, Shanghai, China). For determination, $100 \mu \mathrm{l} /$ well ATP assay buffer was added to a white flat-bottom 96 -well plate. After 3-5 minutes, $20 \mu \mathrm{l}$ of ATP standard or sample was added to each well. The relative light unit was read after at least 2 seconds with a BioTek (Winooski, VT) Synergy4 Multimode Plate Reader.

Xenograft Models. Panc- 1 cells resuspended with PBS $\left(1 \times 10^{7} / \mathrm{ml}\right)$ were injected into subcutaneous tissue of the right axillary fossa $(0.1 \mathrm{ml} /$ animal $)$. After the tumor volumes reached about $0.05 \mathrm{~cm}^{3}$, the animals were given vehicle (PEG400) or CAI ( $30 \mathrm{mg} / \mathrm{kg})$ intragastrically (i.g.) daily for 40 days. In another experiment, after the Panc-1 tumor volumes reached about $1 \mathrm{~cm}^{3}$, the animals were randomly divided into four groups, with six animals in each group (day 0). Vehicle (PEG400); gemcitabine (GEM; $80 \mathrm{mg} / \mathrm{kg})$; a combination of GEM $(80 \mathrm{mg} / \mathrm{kg})$ and CAI $(30 \mathrm{mg} / \mathrm{kg})$; or a combination of GEM $(80 \mathrm{mg} / \mathrm{kg})$, CAI $(30 \mathrm{mg} / \mathrm{kg})$, and 2-DG ( $450 \mathrm{mg} / \mathrm{kg}$ ) was administered for 2 weeks (i.g. daily for PEG400 and CAI; i.p. twice a week for GEM; i.p. daily for 2-DG). The tumor length and width were measured with a sliding caliper on days $0,7,28$, and 35 . Tumor volumes $\left(\right.$ in $\mathrm{cm}^{3}$ ) were calculated as length $\times$ width ${ }^{2} \times 0.5$. Pan-02 cells resuspended with PBS $(5 \times 106 / \mathrm{ml})$ were injected into subcutaneous tissue of the right axillary fossa $(0.1 \mathrm{ml} /$ animal $)$. After the tumor volumes reached $0.05 \mathrm{~cm}^{3}$, the animals were randomly divided and treated with the indicated drugs. Vehicle (PEG400), CAI (30 mg/kg), 2-DG (450 mg/kg), or a combination of CAI and 2-DG was administered (i.g. daily for PEG400 and CAI; i.p. daily for 2-DG). The tumor length and width were measured with a sliding caliper on days 0,9 , and 40 . Tumor volumes (in $\mathrm{cm}^{3}$ ) were calculated as length $\times$ width $^{2} \times 0.5$.

Statistical Analysis. All statistical analyses were performed using GraphPad Prism 6.01 Software (GraphPad Software, La Jolla, CA). The data are presented as the mean \pm S.D. of at least three independent experiments. Statistical analyses were performed using the unpaired Student's $t$ test or one-way analysis of variance followed by Tukey's test. The differences were significant at $P<0.05$.

\section{Results}

CAI Inhibited Mitochondrial Respiration in Panc-1 Cells. During the Seahorse XF analysis of energy metabolism, we added CAI to Port A in the sensor cartridge and found that CAI dose-dependently reduced mitochondrial oxygen consumption in Panc-1 cells within 10 minutes. After the addition of drug oligomycin in Port B, the OCR of each group was further decreased. After the addition of drug FCCP in Port $\mathrm{C}$, the OCR of each group was restored to the level before oligomycin was added. After the addition of the Port D drugs, antimycin and rotenone, the OCR of each group was decreased to a lower level than that of CAI treatment alone (Fig. 1A). Without glutamine supplementation, CAI significantly downregulated the $\mathrm{NAD}+/ \mathrm{NADH}$ ratio in Panc-1 cells (Fig. 1B). These results indicate that CAI can rapidly target mitochondrial respiration in Panc-1 cells, but the mechanism is different from that of oligomycin, since its effect is not affected by the uncoupling agent FCCP. CAI $10 \mu \mathrm{M}$ (in vivo achievable concentration) for 24 hours completely inhibited mitochondrial respiration in Panc-1 cells with an effect comparable to antimycin and rotenone (Fig. 1C). The protein expression of two important subunits, NDUFS7 and NDUFV3, in ETC complex I was compensatorily increased when the ETC activity was greatly inhibited by CAI (Fig. 1D).

CAI Promoted Compensatory Glycolysis and Reductive Carboxylation in Panc-1 Cells. When OXPHOS is defective or inhibited, the cells are supplied with energy, and tricarboxylic acid intermediates by enhancing glycolysis and reductive carboxylation. During the Seahorse XF analysis of energy metabolism, we found that CAI could increase the ECAR of the panc- 1 cells dose-dependently within 10 minutes (Fig. 2A). ECAR test medium contained a sufficient amount of glutamine but no glucose, indicating that CAI promoted the metabolism of glutamine to lactic acid. The live cell count assays showed that glutamine supplementation attenuated the inhibitory effect of CAI on Panc-1 cell viability (Fig. 2B). Glutamine is an important source of $\alpha$-ketoglutarate $(\alpha-\mathrm{KG})$ involved in reductive carboxylation. We examined the effects of CAI on the expression of IDH1 and IDH2 in Panc-1 cells with sufficient supplementation of glutamine. The expression changes suggested that the reductive carboxylation of $\alpha$-KG to generate citrate was enhanced to support cancer cell growth when OXPHOS was inhibited by CAI (Fig. 2C).

Removal of Glutamine Enhanced the Efficacy of CAI, Leading to Intracellular Aspartate Decrease and Autophagy Flux Blocking. Studies have shown that the mitochondrial electron transport chain not only provides energy, but also supports aspartate synthesis in proliferating cells. In addition, electron transport chain defects will directly lead to the block of autophagy flux. These effects are all associated with the regulation of $\mathrm{NAD}+/ \mathrm{NADH}$ by the electron transport chain. As CAI causes NAD+/NADH imbalance in the absence of glutamine, we tested the effects of CAI on aspartate level and autophagy flux. Addition of the electron acceptor pyruvate partially reversed the anticancer effect of CAI, but only in the absence of glutamine (Fig. 3A). In the following experiment, we examined the effect of another electron acceptor, $\alpha$-ketobutyrate (AKB), on the anticancer effect of CAI in the absence of glutamine. AKB significantly attenuated the anticancer effect of CAI or the classic complex I inhibitor rotenone, with a significant recovery of aspartate content (Fig. 3, B and C). In addition, significant accumulation of LC3B and p62 in Panc-1 cells was observed after CAI treatment, but only in the absence of glutamine (Fig. 3D). We used the GFP-RFP-LC3 adenovirus construct to further confirm the block of autolysosome formation by CAI. When an autophagosome fuses with a lysosome to form an autolysosome, the GFP moiety degrades 
A

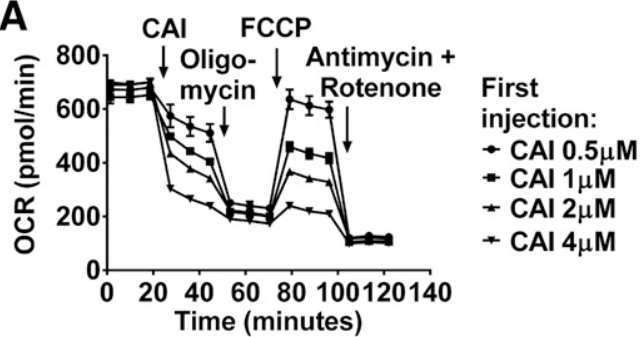

C

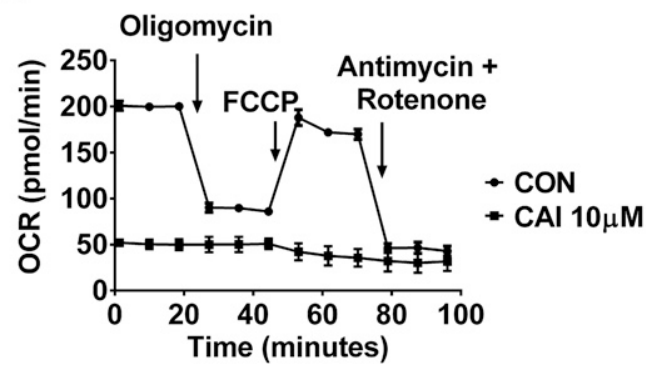

B

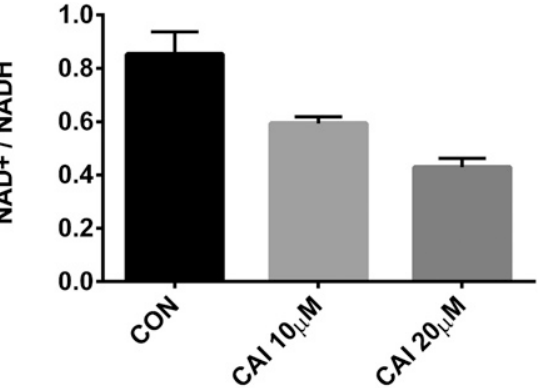

D

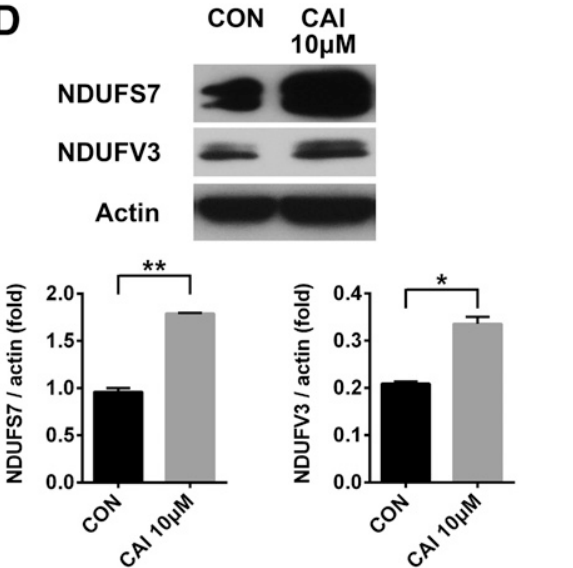

Fig. 1. Effects of CAI on mitochondrial respiration in Panc-1 cells. (A) OCRs were measured under basal conditions and in response to CAI, oligomycin, FCCP, and rotenone plus antimycin $\mathrm{A}$ at the indicated doses in Panc-1 cells $(n=5)$. (B) $\mathrm{NAD}+$ /NADH ratio in Panc-1 cells after CAI treatment for 24 hours $(n=4)$. (C) OCRs were measured under basal conditions and in response to oligomycin, FCCP, and rotenone plus antimycin A at the indicated doses in Panc-1 cells treated with $10 \mu \mathrm{M}$ CAI for 24 hours $(n=5)$. (D) Protein expression of two subunits of mitochondrial electron transport chain complex I in Panc-1 cells treated with vehicle or $10 \mu \mathrm{M}$ CAI for 48 hours. All data are presented as the mean \pm S.D. $* P<0.05 ; * * P<0.01$. CON, vehicle control. from the tandem protein, but RFP-LC3 maintains the puncta. As shown in Fig. 3E, there were more yellow puncta in cells treated with CAI, indicating less GFP moiety degradation and, thus, less autolysosome formation. In addition, we cotreated Panc-1 cells with CAI and chloroquine, and performed the western blotting analysis on lysates of Panc- 1 cells. We found both CAI and chloroquine could increase the protein level of LC3 and p62, but the combination of CAI and chloroquine did not increase LC3 or p62 to a higher level, indicating that CAI might inhibit the autophagy flux instead of promoting autophagosome formation (Fig. 3F).
Glycolysis Inhibitors Enhanced the Anticancer Efficacy of CAI to Cause Energy Depletion. Based on the aforementioned results, we can see that some extracellular nutrients or metabolites, such as glutamine and pyruvate, can influence the anticancer efficacy of CAI, indicating that the effect of CAI in vivo will, therefore, be unstable. We attempted to combine CAI with drugs interfering with other metabolic pathways to enhance anticancer efficacy. We first combined CAI with the lactate dehydrogenase inhibitor OXA and found that the combination had a significant inhibitory effect on the viability of Panc-1 cells cultured in vitro, which was not
A

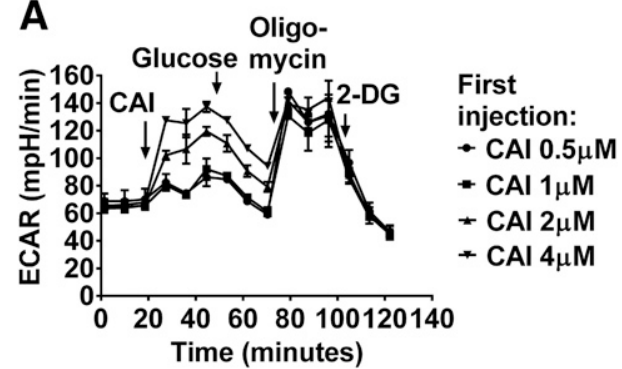

C

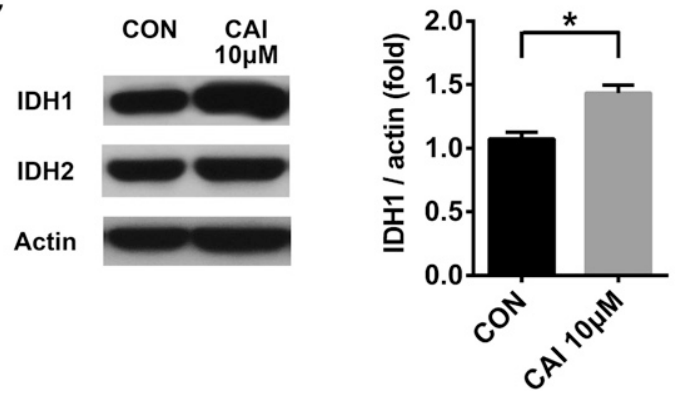

B
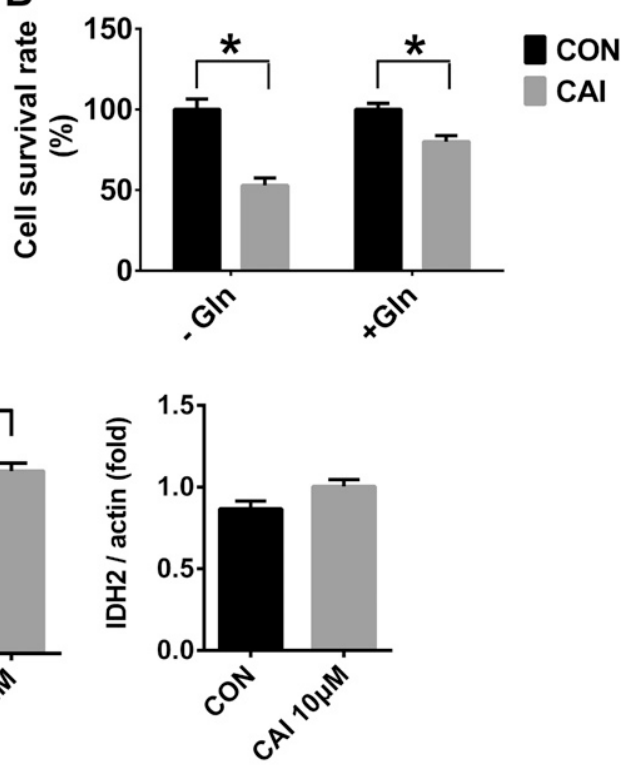

Fig. 2. Effects of CAI on glycolysis and reductive carboxylation in Panc-1 cells. (A) ECARs were measured under basal conditions (without glucose) and in response to CAI, glucose, oligomycin, and 2-DG at the indicated doses in Panc-1 cells $(n=5)$. (B) Glutamine supplementation partially reversed the inhibitory effect of CAI on Panc-1 cell viability ( $n=$ 4). (C) Protein expression of IDH1 and IDH2 in Panc-1 cells treated with vehicle or $10 \mu \mathrm{M}$ CAI for 48 hours. $* P<0.05$. CON, vehicle control; Gln, glutamine. 

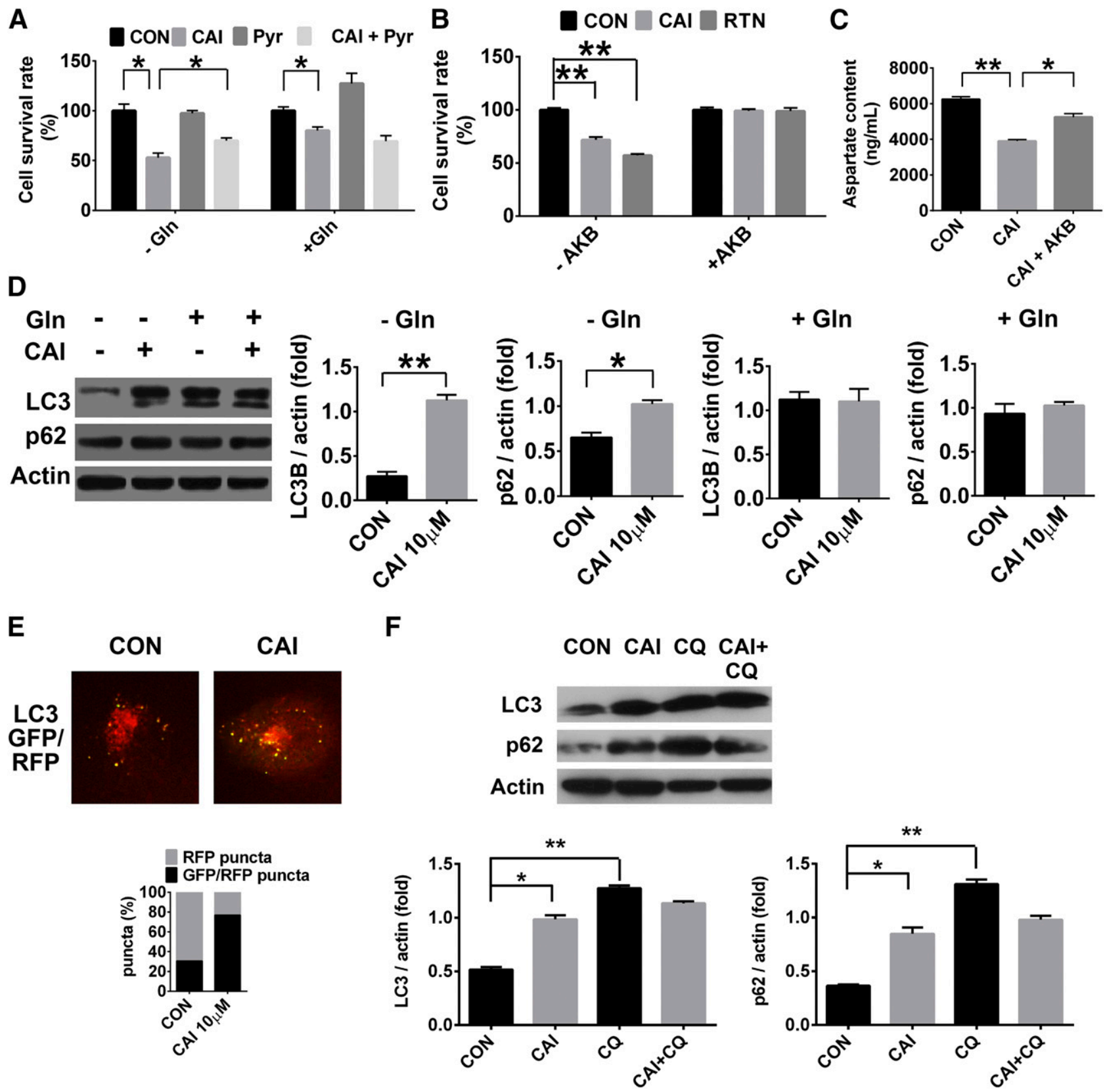

Fig. 3. Anticancer actions of CAI when glutamine supply was limited. (A) Addition of the electron acceptor pyruvate impaired the antitumor effect of CAI, but only in the absence of glutamine $(n=4)$. (B) AKB attenuated the antitumor effect of CAI and rotenone in the absence of glutamine $(n=4)$. (C) Effects of CAI and AKB on aspartate content in Panc-1 cells. (D) CAI caused significant accumulation of LC3B and p62 in Panc-1 cells in the absence of glutamine $(n=3)$. (E) CAI increased yellow puncta in Panc-1 cells transfected with GFP-RFP-LC3 adenovirus. (F) Protein expression of LC3B and p62 in Panc-1 cells treated with CAI and/or chloroquine. All data are presented as the mean \pm S.D. $* P<0.05 ; * * P<0.01$. CON, vehicle control; CQ, chloroquine; Gln, glutamine; Pyr, pyruvate; RTN, rotenone.

affected by glutamine supplementation but was partially attenuated by pyruvate. When glutamine was adequate, the ATP content was decreased to a lower level by the combination compared with CAI or OXA alone, and pyruvate, as the substrate of lactate dehydrogenase, partially reversed this effect (Fig. 4A). Another attempt was to combine CAI with hexokinase inhibitor 2-DG. The combination of CAI and 2-DG had a significant inhibitory effect on the viability of Panc-1 cells cultured in vitro; the anticancer effect was not affected by glutamine or pyruvate supplementation. In the case of adequate glutamine, changes in intracellular ATP content were also consistent with changes in cell viability (Fig. 4B).
The Significant Effect of CAI Plus 2-DG Treatment on Panc-1 In Vivo Growth after Chemotherapy. Although the combination of CAI and 2-DG greatly impaired cancer cells' survival in vitro, we previously found that the efficacy of CAI alone or combined with 2-DG was poor in a C57BL/6 mouse model of pancreatic cancer (Fig. 5A). CAI alone did not inhibit the Panc-1 in vivo growth in a 40-day treatment period (Fig. 5B). However, we found that cotreatment with CAI and 2-DG was of great value when combined with gemcitabine, which significantly delayed tumor progression compared with the GEM-alone group, indicating that drugs disturbing cell metabolism can be useful to enhance the 
A
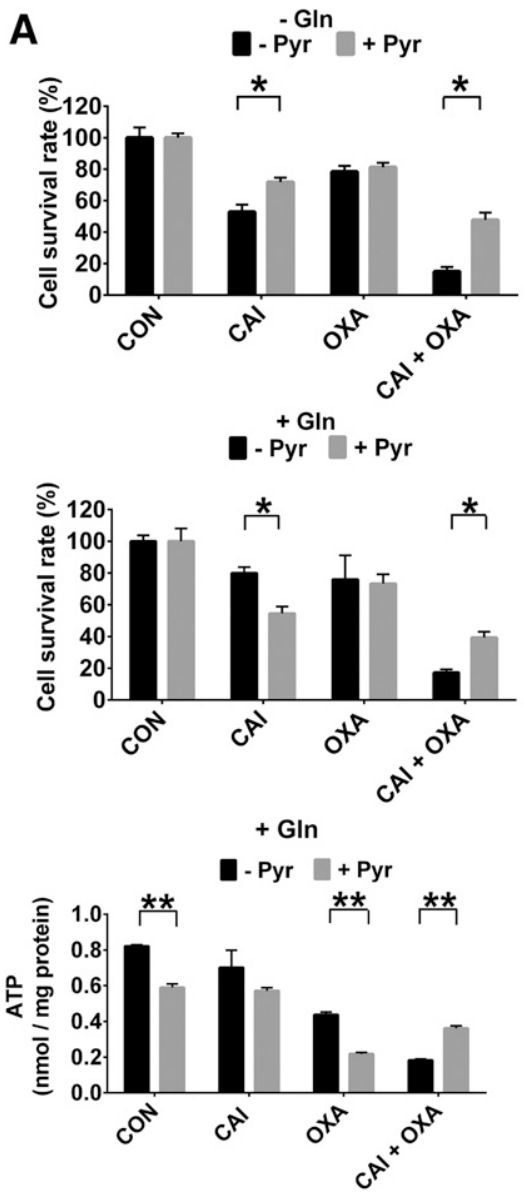

B
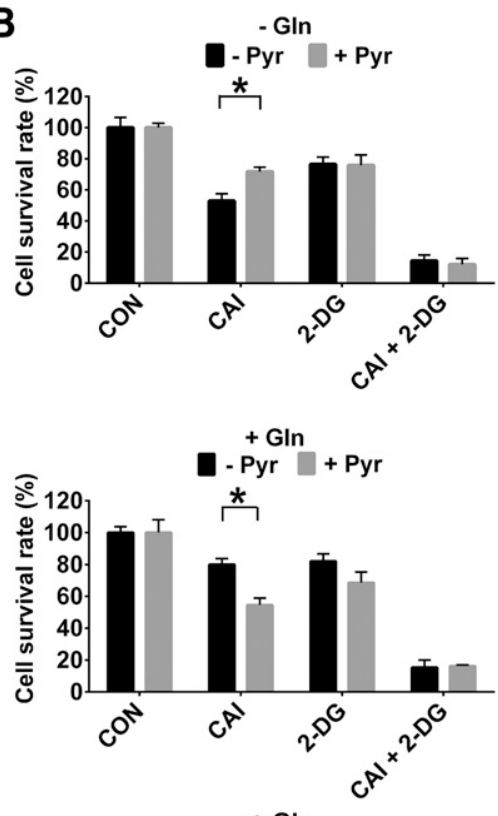

+ GIn

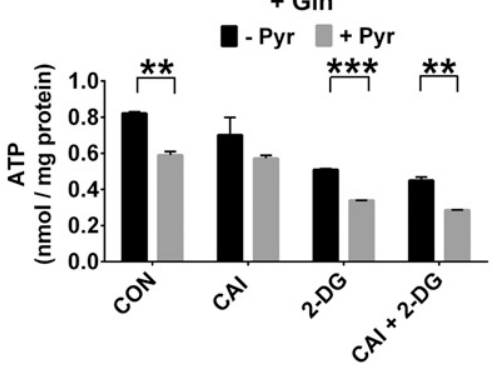

Fig. 4. Effects of the combination of CAI and glycolysis inhibitors on Panc-1 cells. (A) Influences of pyruvate or glutamine on the anticancer effects of the combination of CAI and OXA. (B) Influences of pyruvate or glutamine on the anticancer effects of the combination of CAI and 2-DG. All data are presented as the mean \pm S.D. $(n=4) . * P<0.05$; $* * P<0.01$; $* * * P<0.001$. CON, vehicle control; Gln, glutamine; Pyr, pyruvate. efficacy of the current therapy for pancreatic cancer (Fig. 5C). Based on the aforementioned results, being used with chemotherapy to prevent chemoresistance and delay tumor relapse may be a rational application of this metabolism-disturbing combination strategy.

\section{Discussion}

In this study, the bioenergetic analysis and gene-expression profile of several key metabolic enzymes suggest that CAI can significantly inhibit the activity of the mitochondrial respiration chain in cancer cells and force the cells to use glycolysis and reductive carboxylation for ATP production or biosynthesis. This is consistent with studies demonstrating that compensatory metabolic pathways can be enhanced to support cancer cell survival when mitochondrial OXPHOS is defective (Mullen et al., 2011; Ward and Thompson, 2012; Du et al., 2016). The accumulation of NADH caused by the inhibition of OXPHOS promotes reductive carboxylation of $\alpha$-KG to produce citrate. Glutamine is an important source of $\alpha-\mathrm{KG}$ involved in reductive carboxylation. In this study, CAI elevated ECAR in a short time in the absence of glucose, indicating that CAI can promote the metabolism of glutamine to lactic acid. The expression changes of IDH1 in Panc-1 cells confirm that CAI can promote the reductive carboxylation of $\alpha$-KG to generate isocitrate and then citrate. Our previous data suggested that CAI could increase the expression of phosphoenolpyruvate carboxykinase (PEPCK) in tumor cells. Oxaloacetate can be catalyzed by PEPCK to generate phosphoenolpyruvate and pyruvate, which can increase lactic acid production in addition to glycolysis. All of these data suggest that CAI causes metabolic reprogramming in cancer cells, and the compensatory metabolic pathways are the backup choices for cancer cells to survive when OXPHOS is inhibited or even abandoned, which explains the unstable anticancer efficacy of CAI when being used alone.

In the past, the energy metabolism of cancer cells had been equated with aerobic glycolysis (Warburg, 1956; Racker, 1972). This recognition has been continually amended and replenished. It is generally accepted that mitochondrial respiration is still an important source of energy and is involved in anabolism, which is necessary for the synthesis of multiple substances in most types of cancer cells (Viale et al., 2015; Pavlova and Thompson, 2016). A growing number of studies have shown that mitochondrial metabolism may be an important target for cancer therapy (Hagland et al., 2007; Weinberg and Chandel, 2015). Inhibiting the electron transport chain not only disrupts energy production in mitochondria, but also unbalances the $\mathrm{NAD}+/ \mathrm{NADH}$ ratio and limits the production of electron acceptors, leading to the decrease of aspartate synthesis and autophagy degradation (Baixauli et al., 2015; Birsoy et al., 2015; Sullivan et al., 2015; Gui et al., 2016). However, the compensatory increase of glutamine-dependent reductive carboxylation can promote $\mathrm{NAD}+$ generation from NADH. In this study, we found the anticancer efficacy of CAI was higher in a glutamine-free environment, causing the decrease of the $\mathrm{NAD}+/ \mathrm{NADH}$ ratio, the reduction of intracellular aspartate, and the blocking of 
A
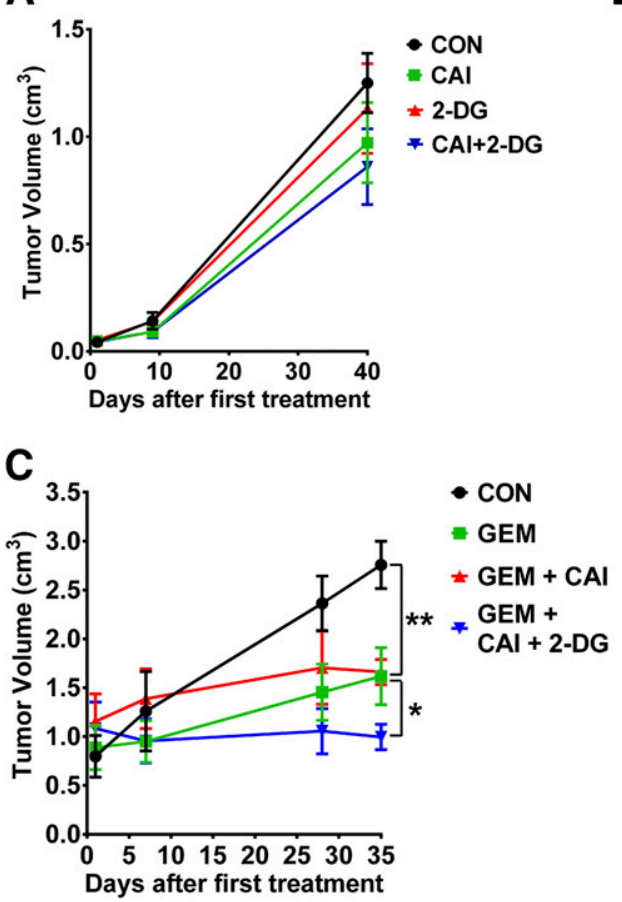
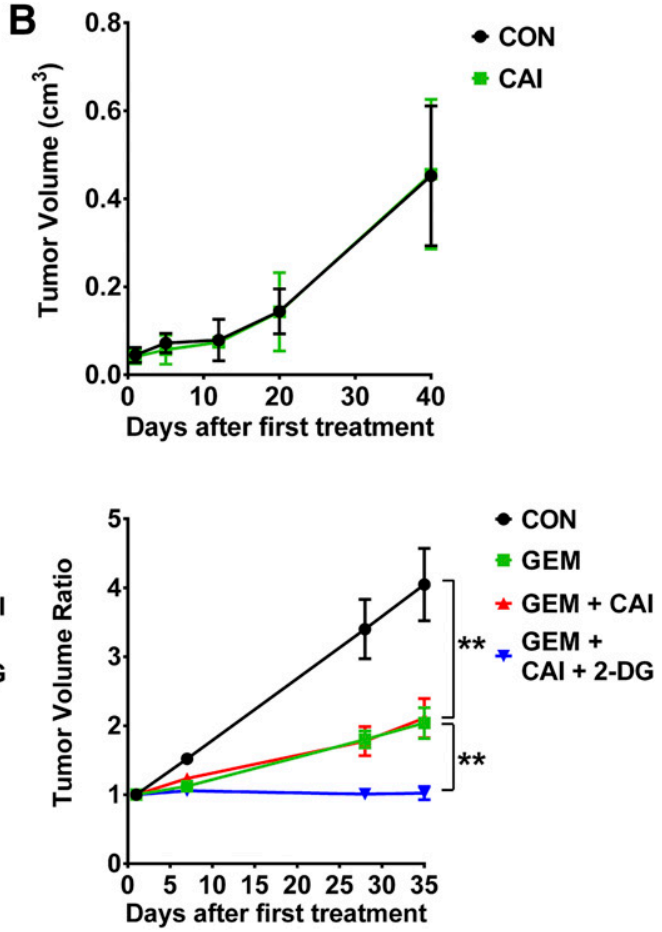

Fig. 5. Effects of the combination of CAI and 2-DG on pancreatic cancer cell in vivo progression. (A) The effects of CAI and/or 2 -DG on Pan-02 in vivo growth $(n=6)$. (B) The effect of CAI on Panc-1 in vivo growth $(n=6)$. (C) The combination of CAI and 2-DG with gemcitabine significantly delayed tumor recurrence compared with gemcitabine alone $(n=6-8)$. The data are presented as the mean \pm S.E.M. $* P<$ $0.05 ; * * P<0.01$. CON, vehicle control. autophagy degradation. In the absence of glutamine, the supplementation of electron acceptors such as pyruvate or AKB attenuated the anticancer efficacy of CAI. We noticed that, when glutamine was adequate, pyruvate could enhance the anticancer efficacy of CAI alone, which was opposite from the effect in the absence of glutamine, suggesting that the accumulation of pyruvate might inhibit the generation of pyruvate from phosphoenolpyruvate and, thus, ATP production (Fig. 6A).

In a glutamine-supplemented environment, the inhibitory effect of CAI on the viability of cancer cells that have been reported previously can also be attributed to its inhibition of mitochondrial respiration. The inhibition of OXPHOS results in a decrease in mitochondrial membrane potential and production of ROS, leading to a decrease in capacity calcium influx and the induction of apoptosis, respectively. Mignen et al. (2005) found that CAI inhibits mitochondrial calcium import, thereby inhibiting capacitative calcium entry and thus affecting cancer cell proliferation. Since mitochondrial membrane potential is the driving force for mitochondrial calcium import, the effect of CAI on the electron transport chain, which downregulates mitochondrial membrane potential, can therefore be considered as an upstream mechanism of its effect on calcium influx. Its apoptosis-inducing effect can be mediated by ROS generated from electron leakage caused by the inhibition of the electron transport chain. In a previous study, we found that the combination of CAI and other drugs that promote ROS production significantly increased the proportion of apoptotic cancer cells (Chen et al., 2017).

As we could see that the extracellular environment can significantly affect the sensitivity of cancer cells to CAI, which may make CAI anticancer efficacy unstable in vivo, we next explored the anticancer efficacy of the combination of CAI and drugs interfering with glycolysis. We found that the combination of CAI and 2-DG markedly increased the anticancer efficacy of each drug, without being affected by the presence of glutamine or pyruvate, owing to the significant decrease of ATP. As 2-DG acted at the upstream step in the process of glycolysis, the anticancer effect of the combination was not affected by the supply of pyruvate (Fig. 6B). The addition of glutamine also failed to reverse the anticancer efficacy of the cotreatment of CAI and 2-DG, which might be attributed to
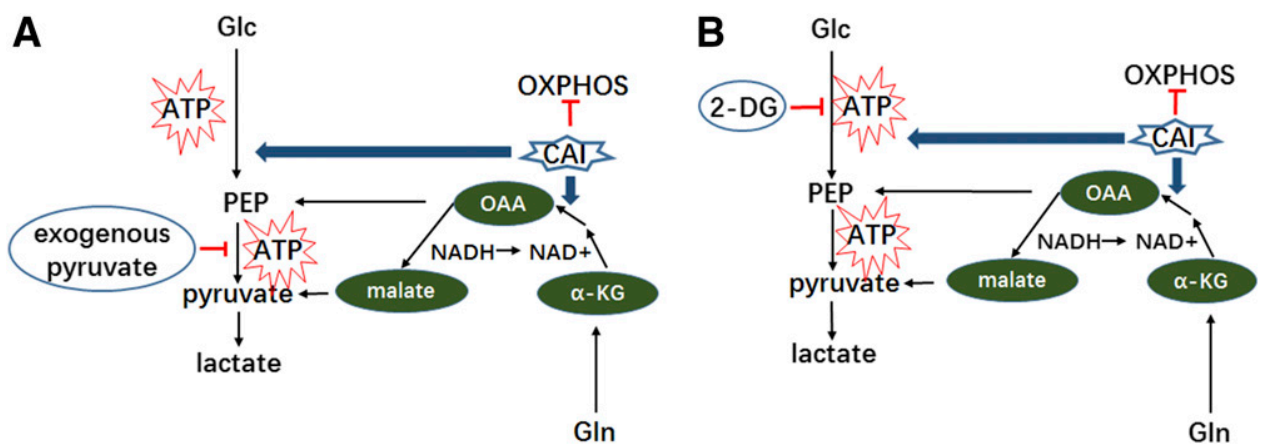

Fig. 6. Intervention of glycolysis synergizes with CAI to block energy production in the presence of glutamine. (A) When glutamine was adequate, exogenous pyruvate influenced ATP production from glycolysis. (B) 2-DG acted at the upstream step in the process of glycolysis and blocked ATP production together with CAI. Glc, glucose; Gln, glutamine; OAA, oxaloacetate; PEP, phosphoenolpyruvate. 
the inhibition of PEPCK or reductive carboxylation by the combination treatment and should be further explored.

We tried to find a rational application of the metabolismdisturbing drugs in cancer therapy. In our previous study, CAI alone or combined with 2-DG only slightly inhibited pancreatic cancer growth in $\mathrm{C} 57 \mathrm{BL} / 6$ mice, suggesting that cancer cells or microenvironments may have other compensatory metabolic pathways in vivo. We next found that the cotreatment of CAI and 2-DG was of great value when combined with gemcitabine, which significantly delayed tumor progression compared with the GEM alone group. Based on the aforementioned results, being used with chemotherapy to prevent chemoresistance and delay tumor relapse may be a rational application of this metabolism-disturbing combination strategy. The reason may be that pancreatic CSCs have been considered to be highly dependent on OXPHOS, and elimination of highly chemoresistant CSCs via inhibition of mitochondrial function (or combined with glycolysis inhibitors) may effectively prevent pancreatic cancer relapse and, thus, improve patients' long-term outcome (Viale et al., 2014; Sancho et al., 2015). For other subpopulations of cancer cells that are highly dependent on mitochondrial metabolism and have compensatory deficits, the effects of CAI are also worth studying.

In conclusion, we demonstrated that inhibiting cancer cell mitochondrial respiration may be the fundamental mechanism of various anticancer actions of CAI. The sensitivity of cancer cells to CAI can be influenced by the metabolic environment. The combination of CAI and 2-DG causes energy depletion in cancer cells, eliminating the rescue effect of the metabolic environment. Inhibiting pancreatic cancer progression after chemotherapy is a rational application of this metabolism-disturbing combination strategy.

\section{Authorship Contributions}

Participated in research design: Ju, Zhang, Guo, Ye.

Conducted experiments: Ju, Fei, S. Li.

Contributed new reagents or analytic tools: Chen, Zhu, J. Li.

Performed data analysis: Ju, Guo, Ye.

Wrote or contributed to the writing of the manuscript: Ju, Guo, Ye.

\section{References}

Ansó E, Weinberg SE, Diebold LP, Thompson BJ, Malinge S, Schumacker PT, Liu X, Zhang Y, Shao Z, Steadman M, et al. (2017) The mitochondrial respiratory chain is essential for haematopoietic stem cell function. Nat Cell Biol 19:614-625.

Baixauli F, Acín-Pérez R, Villarroya-Beltrí C, Mazzeo C, Nuñez-Andrade N, Gabandé-Rodriguez E, Ledesma MD, Blázquez A, Martin MA, Falcón-Pérez JM, et al. (2015) Mitochondrial respiration controls lysosomal function during inflammatory T cell responses. Cell Metab 22:485-498.

Ben Sahra I, Laurent K, Giuliano S, Larbret F, Ponzio G, Gounon P, Le MarchandBrustel Y, Giorgetti-Peraldi S, Cormont M, Bertolotto C, et al. (2010) Targeting cancer cell metabolism: the combination of metformin and 2-deoxyglucose induces p53-dependent apoptosis in prostate cancer cells. Cancer Res 70:2465-2475.

Birsoy K, Wang T, Chen WW, Freinkman E, Abu-Remaileh M, and Sabatini DM (2015) An essential role of the mitochondrial electron transport chain in cell proliferation is to enable aspartate synthesis. Cell 162:540-551.

Burris HA, III, Moore MJ, Andersen J, Green MR, Rothenberg ML, Modiano MR, Cripps MC, Portenoy RK, Storniolo AM, Tarassoff P, et al. (1997) Improvements in survival and clinical benefit with gemcitabine as first-line therapy for patients with advanced pancreas cancer: a randomized trial. J Clin Oncol 15:2403-2413.

Caino MC, Ghosh JC, Chae YC, Vaira V, Rivadeneira DB, Faversani A, Rampini P, Kossenkov AV, Aird KM, Zhang R, et al. (2015) PI3K therapy reprograms mitochondrial trafficking to fuel tumor cell invasion. Proc Natl Acad Sci USA 112 : 8638-8643

Chen C, Ju R, Shi J, Chen W, Sun F, Zhu L, Li J, Zhang D, Ye C, and Guo L (2017) Carboxyamidotriazole synergizes with sorafenib to combat non-small cell lung cancer through inhibition of NANOG and aggravation of apoptosis. J Pharmacol Exp Ther 362:219-229.

Cheong JH, Park ES, Liang J, Dennison JB, Tsavachidou D, Nguyen-Charles C, Wa Cheng K, Hall H, Zhang D, Lu Y, et al. (2011) Dual inhibition of tumor energy pathway by 2-deoxyglucose and metformin is effective against a broad spectrum of preclinical cancer models. Mol Cancer Ther 10:2350-2362.

Corrado C, Raimondo S, Flugy AM, Fontana S, Santoro A, Stassi G, Marfia A, Iovino F, Arlinghaus R, Kohn EC, et al. (2011) Carboxyamidotriazole inhibits cell growth of imatinib-resistant chronic myeloid leukaemia cells including T315I Bcr-Abl mutant by a redox-mediated mechanism. Cancer Lett 300:205-214.

Du J, Yanagida A, Knight K, Engel AL, Vo AH, Jankowski C, Sadilek M, Tran VT, Manson MA, Ramakrishnan A, et al. (2016) Reductive carboxylation is a major metabolic pathway in the retinal pigment epithelium. Proc Natl Acad Sci USA 113: 14710-14715.

Felder CC, Ma AL, Liotta LA, and Kohn EC (1991) The antiproliferative and antimetastatic compound L651582 inhibits muscarinic acetylcholine receptorstimulated calcium influx and arachidonic acid release. J Pharmacol Exp Ther 257: 967-971.

Frattini V, Pagnotta SM, Tala, Fan JJ, Russo MV, Lee SB, Garofano L, Zhang J, Shi P, Lewis G, et al. (2018) A metabolic function of FGFR3-TACC3 gene fusions in cancer. Nature 553:222-227.

Gui DY, Sullivan LB, Luengo A, Hosios AM, Bush LN, Gitego N, Davidson SM, Freinkman E, Thomas CJ, and Vander Heiden MG (2016) Environment dictates dependence on mitochondrial complex I for NAD + and aspartate production and determines cancer cell sensitivity to metformin. Cell Metab 24: $716-727$.

Hagland H, Nikolaisen J, Hodneland LI, Gjertsen BT, Bruserud Ø, and Tronstad KJ (2007) Targeting mitochondria in the treatment of human cancer: a coordinated attack against cancer cell energy metabolism and signalling. Expert Opin Ther Targets 11:1055-1069.

Ju R, Guo L, Li J, Zhu L, Yu X, Chen C, Chen W, Ye C, and Zhang D (2016) Carboxyamidotriazole inhibits oxidative phosphorylation in cancer cells and exerts synergistic anti-cancer effect with glycolysis inhibition. Cancer Lett 370: $232-241$

Kohn EC, Sandeen MA, and Liotta LA (1992) In vivo efficacy of a novel inhibitor of selected signal transduction pathways including calcium, arachidonate, and inositol phosphates. Cancer Res 52:3208-3212.

LeBleu VS, O'Connell JT, Gonzalez Herrera KN, Wikman H, Pantel K, Haigis MC, de Carvalho FM, Damascena A, Domingos Chinen LT, Rocha RM, et al. (2014) PGC$1 \alpha$ mediates mitochondrial biogenesis and oxidative phosphorylation in cancer cells to promote metastasis. Nat Cell Biol 16:992-1003, 1-15.

Louvet C, Labianca R, Hammel P, Lledo G, Zampino MG, André T, Zaniboni A, Ducreux M, Aitini E, Taïeb J, et al.; GERCOR; GISCAD (2005) Gemcitabine in combination with oxaliplatin compared with gemcitabine alone in locally advanced or metastatic pancreatic cancer: results of a GERCOR and GISCAD phase III trial. J Clin Oncol 23:3509-3516.

Luzzi KJ, Varghese HJ, MacDonald IC, Schmidt EE, Kohn EC, Morris VL, Marshall $\mathrm{KE}$, Chambers AF, and Groom AC (1998) Inhibition of angiogenesis in liver metastases by carboxyamidotriazole (CAI). Angiogenesis 2:373-379.

Mignen O, Brink C, Enfissi A, Nadkarni A, Shuttleworth TJ, Giovannucci DR, and Capiod T (2005) Carboxyamidotriazole-induced inhibition of mitochondrial calcium import blocks capacitative calcium entry and cell proliferation in HEK-293 cells. J Cell Sci 118:5615-5623.

Mullen AR, Wheaton WW, Jin ES, Chen PH, Sullivan LB, Cheng T, Yang Y, Linehan WM, Chandel NS, and DeBerardinis RJ (2011) Reductive carboxylation supports growth in tumour cells with defective mitochondria. Nature 481:385-388.

Pavlova NN and Thompson CB (2016) The emerging hallmarks of cancer metabolism. Cell Metab 23:27-47.

Racker E (1972) Bioenergetics and the problem of tumor growth. Am Sci 60:56-63.

Sancho P, Burgos-Ramos E, Tavera A, Bou Kheir T, Jagust P, Schoenhals M, Barneda D, Sellers K, Campos-Olivas R, Graña O, et al. (2015) MYC-PGC-1o balance determines the metabolic phenotype and plasticity of pancreatic cancer stem cells. Cell Metab 22:590-605.

Shewach DS and Lawrence TS (1996) Gemcitabine and radiosensitization in human tumor cells. Invest New Drugs 14:257-263.

Sullivan LB, Gui DY, Hosios AM, Bush LN, Freinkman E, and Vander Heiden MG (2015) Supporting aspartate biosynthesis is an essential function of respiration in proliferating cells. Cell 162:552-563.

Viale A, Corti D, and Draetta GF (2015) Tumors and mitochondrial respiration: a neglected connection. Cancer Res 75:3685-3686.

Viale A, Pettazzoni P, Lyssiotis CA, Ying H, Sánchez N, Marchesini M, Carugo A, Green T, Seth S, Giuliani V, et al. (2014) Oncogene ablation-resistant pancreatic cancer cells depend on mitochondrial function. Nature 514:628-632.

Warburg O (1956) On respiratory impairment in cancer cells. Science 124:269-270.

Ward PS and Thompson CB (2012) Metabolic reprogramming: a cancer hallmark even warburg did not anticipate. Cancer Cell 21:297-308.

Wasilenko WJ, Palad AJ, Somers KD, Blackmore PF, Kohn EC, Rhim JS, Wright GL Jr, and Schellhammer PF (1996) Effects of the calcium influx inhibitor carboxyamido-triazole on the proliferation and invasiveness of human prostate tumor cell lines. Int J Cancer 68:259-264.

Weinberg SE and Chandel NS (2015) Targeting mitochondria metabolism for cancer therapy. Nat Chem Biol 11:9-15.

Address correspondence to: Dr. Caiying Ye, Department of Pharmacology, Institute of Basic Medical Sciences Chinese Academy of Medical Sciences, School of Basic Medicine Peking Union Medical College, Dongdan Santiao 5, Beijing 100005, China. E-mail: caiyingyepumc@163.com; or Dr. Lei Guo, Department of Pharmacology, Institute of Basic Medical Sciences Chinese Academy of Medical Sciences, School of Basic Medicine Peking Union Medical College, Dongdan Santiao 5, Beijing 100005, China. E-mail: guoleistu@126.com 